Berkeley, CA 94720, USA. Internet: lvolkman@nature.berkeley.edu

Received 26 January 1998; accepted 27 March 1998.

Louis G. Hom and Loy E. Volkman

University of California

Berkeley, CA, USA

\section{Nickel-Induced Oligomerization of Proteins Containing 10-Histidine Tags}

\section{BioTechniques 25:20-22 (July 1998)}

Immobilized metal ion affinity chromatography (IMAC) is an efficient method of protein purification. Several systems for protein expression and purification in which a recombinant protein is fused to an $\mathrm{N}$ - or C-terminal oligohistidine tag are commercially available. The tag of six to ten histidine residues binds with high affinity to metal-chelate complexes and allows for the rapid and facile purification of the fusion protein. Elution is carried out by lowering the $\mathrm{pH}$ or by the addition of competing metal ligands such as imidazole or EDTA (6).

We have used IMAC to purify a homeodomain-containing protein for structural studies by nuclear magnetic resonance (NMR) spectroscopy. NMR requires rapid purification of micromole quantities of protein in good yield. The low molecular weight of the affinity tag and its ability to work under both native and denaturing conditions makes the polyhistidine tag IMAC the method of choice for purifying isotopically labeled proteins.

The pre-B-cell leukemia transcription factor $1(P B X 1)$ gene was initially identified because of its involvement in some pre-B-cell leukemias $(1,3)$. We have produced a fusion protein, $\mathrm{Pbx}$ 233-319, using the pET vector system (Novagen, Madison, WI, USA), in which amino acids 233-319 of PBX are fused to an N-terminal ten-histidine tag by a Factor Xa cleavage site. The 108 amino acid (12560 Da) protein, expressed in $E$. coli strain BL21 $(\lambda \mathrm{DE} 3)$, is purified on $\mathrm{Ni}^{2+}$-charged Chelating Sepharose ${ }^{\circledR}$ Fast Flow (Amersham Pharmacia Biotech, Uppsala, Sweden) with a yield of approximately $40 \mathrm{mg}$ of recombinant protein per liter of cell culture.

Initial attempts at purifying $\mathrm{Pbx} 233$ 319 resulted in the apparent co-purification of a protein migrating at $41 \mathrm{kDa}$ in sodium dodecyl sulfate polyacrylamide gel electrophoresis (SDS-PAGE). Samples incubated in elution buffer $(1 \mathrm{M}$ imidazole, $0.5 \mathrm{M} \mathrm{NaCl}, 20 \mathrm{mM}$ Tris$\mathrm{HCl}, \mathrm{pH}$ 7.9) containing $6 \mathrm{M}$ urea at $4^{\circ} \mathrm{C}$ for several days displayed a ladder of higher-molecular-weight proteins leading to the conclusion that $\mathrm{Pbx} 233$ 319 had oligomerized.

This conclusion was confirmed by the reversible formation and dissociation of the oligomers by $\mathrm{Ni}^{2+}$ and EDTA. As shown in Figure 1, addition of EDTA converted the 41-kDa protein into monomeric Pbx233-319. Conversely, monomeric Pbx could be oligomerized to a higher-mass species by the addition of $10 \mathrm{mM}$ nickel sulfate. In addition, it was found that while heating for a short period ( $<1 \mathrm{~min}$ ) did not disrupt oligomerization, $\mathrm{Pbx} 233-319$ could be returned to the monomeric form by vigorous boiling in loading buffer. We conclude that the polyhistidine affinity tag forms reversibly crosslinked oligomers that are resistant to $1 \%$ SDS, $6 \mathrm{M}$ urea and $10 \mathrm{mM}$ dithiothreitol (DTT). In samples without added nickel, we believe that sufficient metal leached off the IMAC affinity column for efficient oligomerization. Removal of the affinity tag by treatment with Factor Xa abolished the ability of $\mathrm{Pbx} 233-319$ to form oligomers (data not shown).

We have also observed precipitation of Pbx233-319 during dialysis following IMAC or when using improperly prepared dialysis tubing. The precipitate dissolved upon addition of EDTA to the dialysis buffer. Similar observations have been reported for His-tag-purified yeast citrate synthase (2) and His-tagged eukaryotic transcription factors (5).

The exact nature of the oligomers is unclear. Pbx233-319 migrates anomo- lously during SDS-PAGE as a $16-\mathrm{kDa}$ band, and disulfide-linked dimers of Pbx233-319 migrate at $30 \mathrm{kDa}$ (data not shown). In contrast, the nickel-induced oligomers showed apparent mobilities of 41 and $85 \mathrm{kDa}$, which suggest oligomer stoichiometries of 3 and 6 , respectively. Aside from its intrinsic interest, understanding the nature of the metal-histidine complexes might be of interest for potential applications in cell surface receptor cross-linking, metaldependent enzyme-linked immunosorbent assays (ELISAs) (4) or metalsensing molecular switches.

We chose to use a ten-histidine affinity tag to avoid problems of poor binding observed with other fusion protein constructs (T. Sprules, unpublished). Six-histidine affinity tags are more prevalent in the literature than ten-histidine tags but have lower affinity for the IMAC columns. The presence of ten histidine residues in our $\mathrm{Pbx}$ fusion protein may favor His-tag oligomerization.

The presence of protein precipitates or oligomers after IMAC is of practical importance for subsequent chromatography. Samples appearing to be mono-

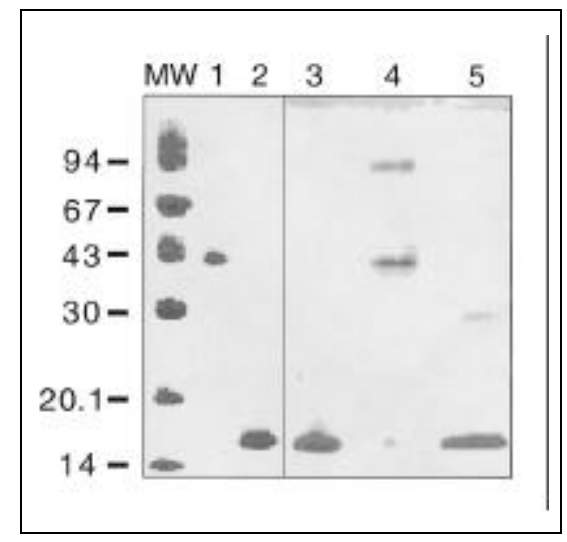

Figure 1. Nickel-induced oligomerization of Pbx233-319. Lane 1: oligomerized Pbx233-319, following purification on $\mathrm{Ni}^{2+}$-charged Chelating Sepharose under denaturing conditions $(6 \mathrm{M}$ urea). Lane 2: previous sample plus $10 \mathrm{mM}$ EDTA. Lane 3: Pbx233-319 dialyzed against 10 mM EDTA immediately following IMAC purification, then dialyzed against EDTA-free buffer. Addition of $10 \mathrm{mM}$ nickel sulfate to the previous sample results in the formation of oligomers (lane 4), which can be reversed by boiling the sample for several minutes (lane 5). Samples were dissolved in SDS loading buffer ( $50 \mathrm{mM}$ Tris- $\mathrm{HCl}$, $10 \%$ glycerol, $2 \%$ SDS, $0.1 \%$ bromophenol blue, $10 \mathrm{mM}$ DTT) prior to electrophoresis on Homogeneous 20 PhastGels ${ }^{\circledR}$ (Amersham Pharmacia Biotech) but not boiled unless indicated. 
meric in SDS-PAGE following boiling in loading buffer might in fact be oligomeric, leading to multiple or broadened peaks in size exclusion or ion-exchange chromatography. Therefore, we recommend adding EDTA to eluted IMAC fractions immediately after collection. Excess EDTA and EDTA-metal complexes can then be removed by dialysis or size-exclusion chromatography.

\section{REFERENCES}

1.Kamps, M.P., C. Murre, X.H. Sun and D. Baltimore. 1990. A new homeobox gene contributes the DNA binding domain of the $\mathrm{t}(1 ; 19)$ translocation protein in pre-B ALL. Cell 60:547-555.

2.Lindner, P., B. Guth, C. Wulfing, C. Krebber, B. Steipe, F. Muller and A. Pluckthun. 1992. Purification of native proteins from the cytoplasm and periplasm of Escherichia coli using IMAC and histidine tails: a comparison of proteins and protocols. Methods: Companion Methods Enzymol. 4:41-56.

3.Nourse, J., D. Mellentin, N. Galili, J. Walkinson, E. Stanbridge, S.D. Smith and M.L. Cleary. 1990. Chromosomal translocation $\mathrm{t}(1 ; 19)$ results in synthesis of a homeobox fusion mRNA that codes for a potential chimeric transcription factor. Cell 60:535-545.

4.Paborsky, L.R., K.E. Dunn, C.S. Gibbs and J.P. Dougherty. 1996. A nickel chelate microtiter plate assay for six histidine-containing proteins. Anal. Biochem. 234:60-65.

5.Van Dyke, M.W., M. Sirito and M. Sawadogo. 1992. Single-step purification of bacterially expressed polypeptides containing an oligohistidine domain. Gene 111:99-104.

6.Winzerling, J.J., P. Berna and J. Porath. 1992. How to use immobilized metal ion affinity chromatography. Methods: Companion Methods Enzymol. 4:4-13.

This work is supported by Canadian Medical Research Council grants to K.G. and M.F., a Hydro-Québec McGill Major Award to T.S. and a Chercheur-Boursier of the Fonds de la Recherche en Santé du Québec to M.F. Address correspondence to Ms. Tara Sprules, Department of Biochemistry, McIntyre Medical Science Building, McGill University, 3655 Drummond, Montreal, QC H3G 1Y6, Canada. Internet: tara@bri.nrc.ca

Received 8 December 1997; accepted 23 March 1998.

Tara Sprules, Nancy Green, Mark Featherstone and Kalle Gehring

McGill University

Montreal, QC, Canada

\section{Expression Vector Containing an N-Termi- nal Epitope Tag for Dictyostelium discoideum}

BioTechniques 25:22-24 (July 1998)

For studies of gene function, an epitope tagging system can provide several advantages. Epitope tagging involves the fusion of a small, well-characterized epitope (usually in the form of a short peptide) with another protein. This method has the obvious advantage of bypassing the requirement for antibodies specifically against the protein of interest. Furthermore, an epitopetagged protein can readily be distinguished from the endogenous protein.

The cellular slime mold Dictyostelium discoideum is a eukaroyte used as a model system to study the processes of development and many aspects of cell biology. To date, several expression vectors for this organism have been designed for epitope tagging on the $\mathrm{C}$ termini of proteins $(1,8)$, but very few $\mathrm{N}$ terminal tags are available $(2,9,12)$. Many proteins, such as ras proteins, do not permit C-terminal tags because posttranslational processings on their $\mathrm{C}$ termini are essential for their function. The green fluorescent protein (GFP) has been used as an N-terminal tag in several Dictyostelium studies $(9,12)$. The application of this GFP tag, however, can be limited by its high molecular weight (9). We describe here the successful application of a new N-terminal epitope-tagged expression vector for studying gene functions in Dictyostelium discoideum.

The peptide used for epitope tagging contained the first 12 amino acids of the phage T7 gene 10 (7). Antibodies to this peptide are commercially available (Novagen, Madison, WI, USA). To construct the vector, we started with the Dictyostelium shuttle vector EXP4(+) (4). It can be easily maintained and amplified in E. coli in the presence of the selective drug ampicillin. The EXP4(+) vector contains the G418-selectable marker, which is used for transforming DNA into the Dictyostelium genome (6). It also contains a well-characterized Dictyostelium constitutive promoter, the actin 15 promoter (3), which has a known expression pattern.

To construct an efficient epitope-

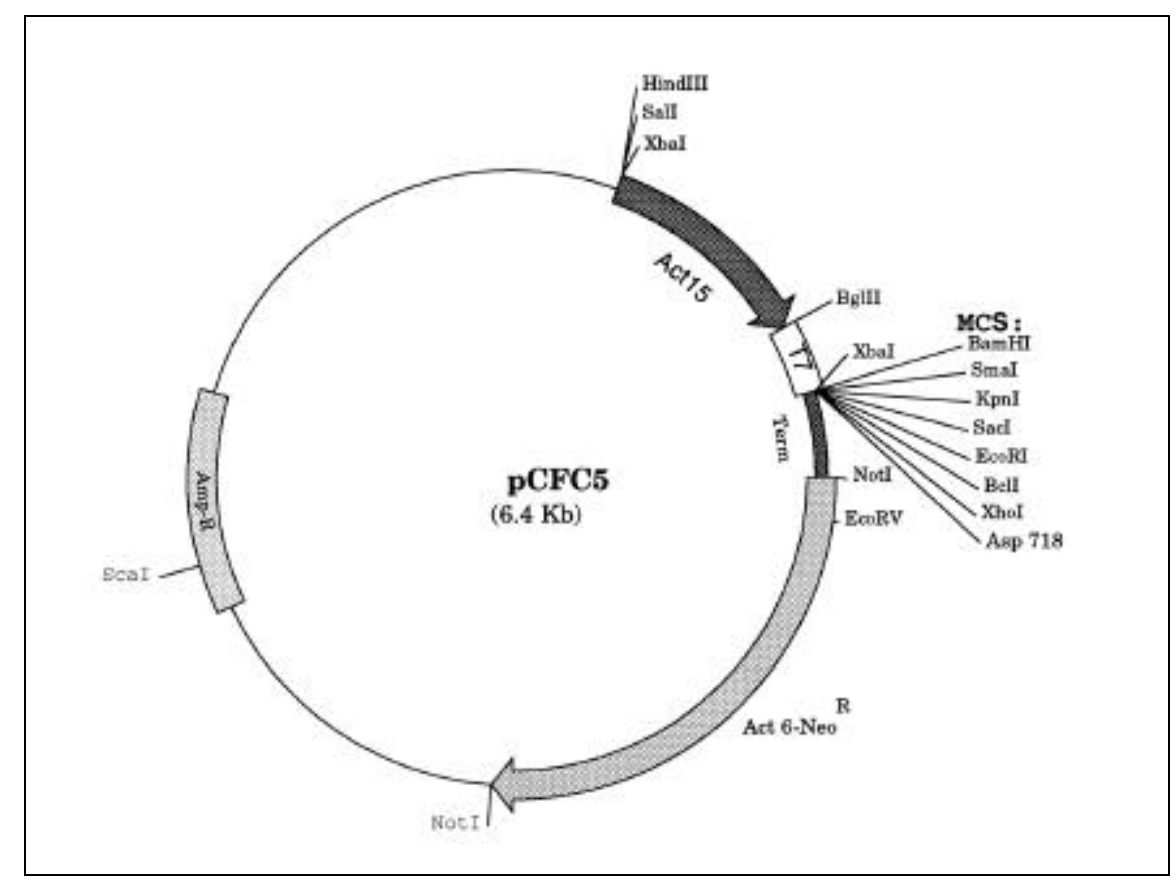

Figure 1. Map of pCFC5 vector used to express T7-tagged proteins in Dictyostelium. The following sequence of the modified T7 epitope tag (with modified nucleotides underlined) includes the sequence immediately surrounding the ATG initiation. 5'-AGATCT AAAAA ATG GCT AGT ATG ACT GGT GGT CAA CAA ATG GGT CGT-3'. 